\title{
Rapid Mission Concept Development at the 2019 Caltech Space Challenge: A Small Lander Network Studying the Habitability of Enceladus
}

Kelsey Doerksen ${ }^{1}$, Katiyayni Balachandran ${ }^{2}$, Paolo Cappuccio ${ }^{3}$, Julia Di ${ }^{4}$, Jared Fuchs ${ }^{5}$, Alessia Gloder ${ }^{6}$, Rebecca Jolitz ${ }^{7}$, Monica $\mathrm{Li}^{8}$, Dan Limonchik ${ }^{9}$, Lotfi Massarweh ${ }^{10,11}$, Atila Meszaros ${ }^{12}$, Daniel Naftalovich ${ }^{13}$, Erica Nathan ${ }^{14}$, Thomas Peev ${ }^{15}$, Marc RoviraNavarro ${ }^{16,17}$, Shreya Santra ${ }^{18}$

${ }^{1}$ University of Western Ontario, ${ }^{2}$ University of Texas at Arlington, ${ }^{3}$ Sapienza University of Rome, ${ }^{4}$ Stanford University, ${ }^{5}$ University of Alabama in Huntsville, ${ }^{6}$ University of Padua, ${ }^{7}$ University of Colorado Boulder, ${ }^{8}$ University of California Berkeley, ${ }^{9}$ Rhode Island School of Design, ${ }^{10}$ Deimos Engenharia S.A, ${ }^{11}$ Instituto Superior Tecnico, ${ }^{12}$ Universidad Peruana Cayetano Heredia, ${ }^{13}$ California Institute of Technology, ${ }^{14}$ Brown University, ${ }^{15}$ University of California Davis, ${ }^{16}$ Delft University of Technology, ${ }^{17} \mathrm{NIOZ}$ Royal Institute for Sea Research, ${ }^{18}$ Tohoku University Japan

${ }^{1}$ kdoerkse@uwo.ca ${ }^{2}$ katiyayni.balachandran@mavs.uta.edu

\begin{abstract}
The 2019 Caltech Space Challenge was a one-week intensive mission proposal challenge that brought an international group of 32 post-secondary students from various disciplines to design multi-lander mission concepts for Enceladus. The students were divided into two competing teams of 16, Team Voyager and Team Explorer. In this paper, Team Voyager describes their process and challenges in conceptualizing the winning mission proposal (SILENUS) of an orbiter and a network of landers. The final mission architecture proposes a mission where the science data return lasts just over one year and sends an orbiting satellite housing science instrumentation to Enceladus, dropping off four penetrating seismometers to the surface of the icy moon. In our paper, we provide an overview of our high-level mission design, an analysis of team structure and dynamics, the resources utilized by the teams to assist with mission conception, as well as the challenges and learning outcomes of the week as a framework for future rapid mission concept development.
\end{abstract}

Keywords-Caltech Space Challenge; Enceladus; Seismometer

\section{INTRODUCTION}

The Caltech Space Challenge brings 32 talented and highly motivated undergraduate and graduate students to participate in a 5-day international space mission design competition. The challenge is a unique opportunity for young and enthusiastic students to build technical and teamwork skills, interact with world-renowned experts in space exploration and connect to like-minded peers from across the globe. The participants are split into two teams of 16 students each, aptly named Team Voyager and Team Explorer. Both teams are mentored by industry experts, as well as researchers from NASA and academia to design their mission concept from scratch. At the end of the week, one winner is selected by a panel of judges from leading aerospace organizations, including NASA, Northrup Grumman, and Lockheed Martin for the 2019 challenge. The winners of the challenge, Team Voyager, present in the remainder of this paper, mission SILENUS: Spectrometer Investigating the Livability of Enceladus with a Network Using Seismometers. The paper is structured as follows: to begin, a justification as to why a mission to
Enceladus was chosen, then an overview of the SILENUS mission from an engineering, science, and systems engineering perspective. Following the SILENUS mission, the team formulation strategy and available resources provided by the Caltech Space Challenge will be detailed. Challenges related to interdisciplinary teamwork and management are discussed, concluding with post-challenge learning outcomes.

\section{BACKGROUND: WhY A Mission to ENCELADUS?}

According to the Planetary Science Decadal Survey in $2011^{1}$, a primary unanswered question is if "beyond Earth [there] are contemporary habitats elsewhere in the solar system with necessary conditions, organic matter, water, energy, and nutrients to sustain life and do organisms live there now?". This question has intrigued mankind for centuries and therefore is not surprising that the search for life in our solar system is one of the drivers for space exploration ${ }^{2}$.

While in the early days of solar system exploration we hoped to find clues to answer the question of extraterrestrial life within the habitable zone, recent evidence of subsurface water reservoirs in the moons of the outer solar system ${ }^{3}$ have shifted our attention to the satellites of gas giants in the outer solar system like Jupiter and Saturn. In particular, Cassini discovered that Saturn's moon Enceladus, an object initially thought to be a geologically dead body is one of the most likely harbours of contemporary extraterrestrial life. Cassini's measurements not only reported evidence for a differentiated interior structure with a subsurface water ocean possibly in contact with the rocky core ${ }^{4}$, it also discovered geyser-like jets in the south polar region of Enceladus, dubbed Tiger Stripes ${ }^{5}$. These jets vent water vapor and solid material from the

\footnotetext{
${ }^{1}$ https://solarsystem.nasa.gov/system/downloadable_items/784_Planetary_Scie nce_Decadal_2013-2022.pdf

2 Background adapted from:

http://www.spacechallenge.caltech.edu/description

${ }^{3}$ Khurana 1998, Postberg et al. 2011

${ }^{4}$ Postberg et al. 2011; Less et al., 2014; Hsu et al. 2015; Thomas et al. 2016 ${ }^{5}$ Porco et al. 2006
} 
interior ocean into space. During close flybys, Cassini's mass spectrometer detected complex organic compounds contained in the plumes. This finding fueled speculation about the presence of life in Enceladus' subsurface ocean, but Cassini's instrumentation was not designed to detect life, leaving this significant question to be answered by follow-on missions.

Ideally, probing Enceladus for the presence of life means accessing not only its plumes, but also the most likely location of indicative biomolecules: the surface orifices of its jets, located in the south polar Tiger Stripes region. Given the incomplete knowledge of Enceladus' surface and its plumes, a classic single-lander mission seems too risky. But what if the risk could be spread among multiple small, cost-effective landers? This was the driving question behind the 2019 Caltech Space Challenge.

\section{SILENUS MISSION}

SILENUS is a New Frontiers class mission proposal that investigates the habitability of Saturn's moon, Enceladus. The programmatic constraints on the mission concept as set by the challenge were the following:

1. Land as close as possible to the plumes' sources

2. Use a collection of small landers/rovers

3. Target a New Frontiers class mission arriving at Enceladus between 2036 and 2042

4. Comply with planetary protection guidelines

5. Launch the mission with one SLS-type launcher (excluding cost of launch)

The proposed mission timeline, as shown in Figure 1, is such that in 2028, the SLS vehicle will be launched from Earth, arriving at Enceladus 13 years later with a series of gravity assists from Venus, Earth, and Saturn.

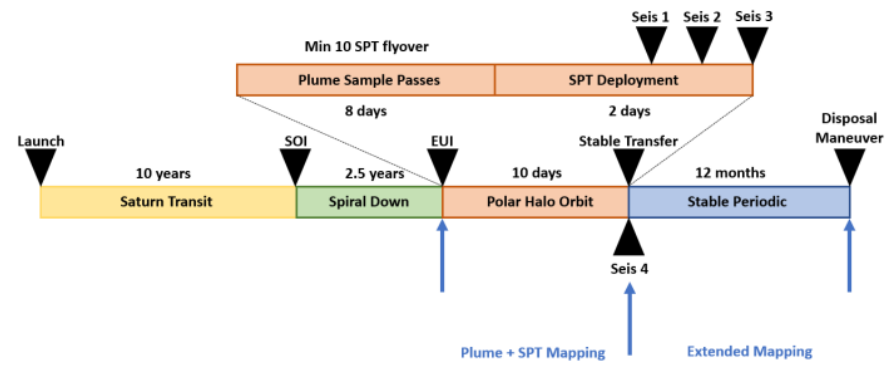

Figure 1: SILENUS Mission Timeline

Together with the orbiter and science payload, SILENUS will enter an orbit around Enceladus where a network of penetrators will be deployed to Samarkand Sulcus and the South Polar Terrain (SPT), the main regions of interest for this mission. The plume ejecta will be investigated with a mass spectrometer onboard the orbiter, and the network of seismometers will be deployed to the surface of Enceladus with the purpose of understanding the tomography. The data collected from this mission will be a transformational advance in understanding more about the geological features of icy moons in our solar system as well as an opportunity for collaborations with academia, industry and the public.

\section{A. Science Objectives \& Instrumentation}

After constructing a science traceability matrix, the following primary scientific objectives were chosen in decreasing order of priority:

1. Characterize the organic chemistry of the plume ejecta;

2. Characterize the inorganic chemistry of the plume ejecta;

3. Constrain the age, structure, and exchange pathways of habitable environments.

In order to achieve these objectives, the cost constraints, hazards, and engineering challenges of surface operations were taken into consideration and a remote-sensing focused mission was selected. This encompassed an Enceladus orbiter with four seismometer penetrators, to be spread across the surface of the moon (one in the North and remaining three in the SPT). Remote science instrumentation included a mass spectrometer, capillary electrophoresis system (CES), an ionselective electrodes wet chemistry lab (ISE), and laser altimeter. For in-situ science, a context camera and seismometer network were chosen to be deployed from the orbiter. The science-selected landing site is shown in Figure 2. The circles represent selected landing sites categorized broadly by hazard level (green is least hazardous, yellow is more hazardous, and orange most hazardous). Circles outlined in white are final selections and black outlines indicate backout landing sites. Selected high resolution Cassini imagery is overlaid.

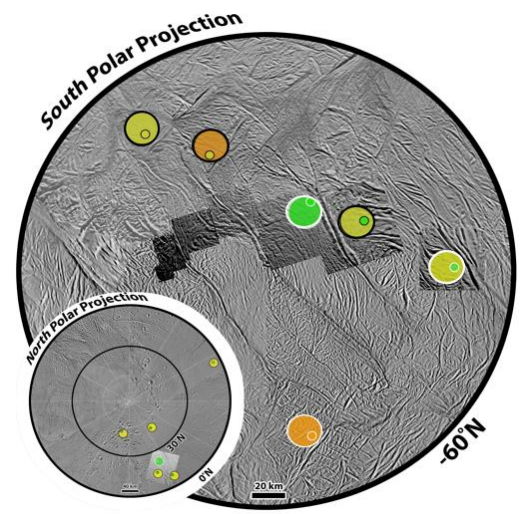

Figure 2: Landing Site Selection

\section{B. Engineering Constraints}

From an engineering perspective, the orbiter was modelled closely after previously successful missions, specifically the Cassini spacecraft. The key difference between Team Voyager's architecture and Cassini, is the use of solar panels as the power source, instead of radioisotope thermoelectric generators (RTGs). Solar panels were ultimately chosen by the team as they provided the same amount of power to the orbiter, with half the cost of an RTG, a crucial decision that factored in heavily with the judges' choice of winning proposal. The penetrators were modelled ballistically-shaped, such that upon impact with Enceladus' surface they would 
successfully penetrate the surface to partially submerge the seismometers housed within. Figure 3 shows the modelled design of the proposed orbiter, and Figure 4 shows the seismometer penetrators (four in total). Flight heritage and technology readiness level (TRL) were key factors for selecting the subsystem architecture and components, as a New Frontiers mission budget does not allow for a large sum towards prototyping and development of technology. The subsystem equipment onboard the orbiter have a TRL between 8-9 (with 9 being the highest), and the penetrator components chosen have a TRL between 3-5, due to the lack of preexisting technology utilized in prior space missions for this purpose.

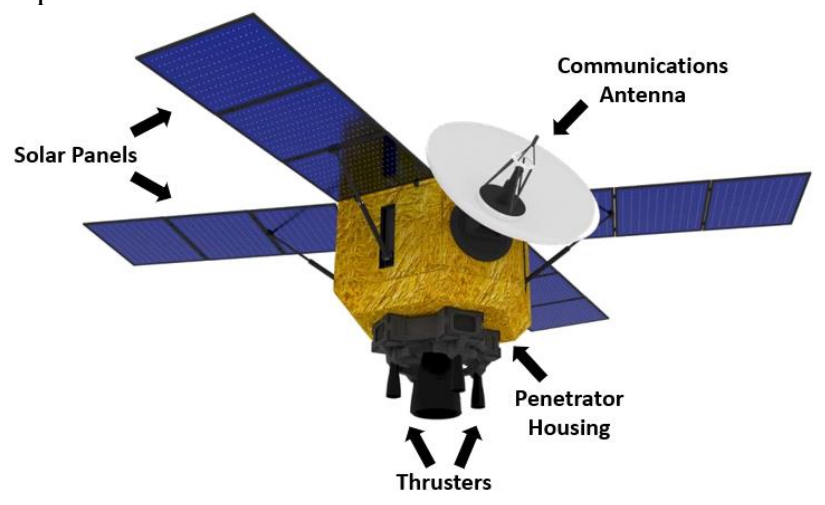

FIG 3: SILENUS Orbiter

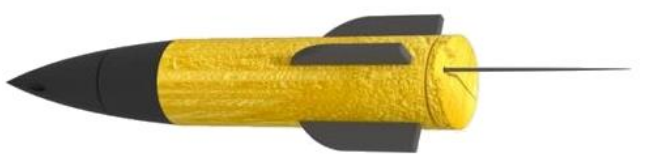

FIG 4: SILENUS Penetrator

\section{Budgets}

The preliminary mass budget for the orbiter and penetrators was done through first-order approximation using Space Mission Engineering: The New SMAD [1] and past reports from Cassini. Sizing to our model and mission constraints resulted in a total mass of $890 \mathrm{~kg}$ for the orbiter and $15 \mathrm{~kg}$ for each penetrator (including all subsystem components). Knowing the feasibility of solar panels and their power generation capabilities $(370 \mathrm{~W})$, allocations were made to the major subsystems to estimate total power needed.

The mission cost budget was prepared assuming no launch costs, as instructed in the challenge, and a 30\% margin on all other costs (following NASA protocol). Based on anonymized past missions and historical data, all output phases A through D using Fiscal Year 16 as a benchmark showed our total costs at $\$ 770$ million, which is well within the budget of $\$ 1$ billion including the reserves. The budget included costs for various items such as project management, safety and mission assurance, mission operating systems and ground data systems with the largest portion set aside for flight systems and payload. Costs for labor and time involved for ground assessment post-launch were also included. Breaking down the flight system costs, excess from the science payload was instrumental in meeting our budget for the orbiter and penetrators. Several major decisions for power generation (i.e. solar panels vs RTGs) were based on the availability of extra funds. All spacecraft component systems such as telemetry \& tracking, attitude determination and control, power, structures, among others were fit into the budget with cross-checks done individually for each subsystem based on science/surveillance mission types and their nominal costs by weight, mass, and power.

\section{Planetary Protection Measures}

Planetary protection is defined as a set of practices to:

1. Not contaminate any planetary bodies outside of Earth

2. Prevent any contamination of Earth from exo-earth material

Following Cassini's discovery of Enceladus's ocean, "the probability of inadvertent contamination of an ocean or other liquid water body" must be reduced to less than $10^{-4}$ per mission (NPR 8020.12D, Sec. 5.4) [2]. For SILENUS to follow planetary protection measures, the orbiter must be destroyed at the end of the mission to avoid any contact with Enceladus or other regions of interest. Drawing from the proposed mission "Testing the Habitability of Enceladus's Ocean" (THEO) [3], we proposed to crash the orbiter into Tethys, a nearby moon that has been previously determined to be inhospitable to life. It was this decision, combined with the self-imposed budgetary constraints set by team Voyager, which the judges deemed enough to grant our team the winning proposal.

\section{MisSION DESIGN WORK AS A TEAM}

\section{A. Team Structure}

Following the introductory session and team building workshop, the teams split to get acquainted with their members. A brainstorming session was used as an icebreaker, where each member listed their expertise and skills they could contribute towards the challenge. This exercise allowed for a preliminary grouping into Science, Engineering and Management sub-teams. As the week progressed, lines of communication were required between the science and engineering sub-teams and the division of work was crystallized. The Science team focused on the science objectives and data collection to be accomplished throughout the mission. The Engineering team designed the means to achieve the science objectives and successfully complete the mission. Each sub-team was assigned a systems engineer, with a third systems member designated to the management and coordination between sub-teams. Systems engineers were also in charge of ensuring the constraints were met and played a pivotal role in project management and completing the deliverables. A designated Team Voyager liaison that relayed information between the team and the challenge co-chairs was also utilized. 


\section{B. Mentorship, Workshops, and Techniques for Concept} Creation

Mentorship was a huge part of the Caltech Space Challenge, and one of the most valuable take-aways for participants. Mentors from academia, past Space Challenge participants, and industry provided feedback on design work and general support to both teams throughout the week. NASA JPL dedicated a significant amount of time to this, as the teams spent the first full day of the Space Challenge at their campus; half the day for teams to learn mission proposal design and collaboration techniques, and the other half as a tour of the facilities. Figure 5 captures an exciting stop on the tour at the NASA JPL Mars Yard with their model of the Curiosity rover. The day acted as both a learning and inspiration opportunity.

The workshops run by JPL included:

1. "Getable" Science Stories: Inspired by Pixar, the concept of storytelling involves six sentences ${ }^{6}$. Pixar's storytelling method can be applied to space missions, by breaking down a mission proposal into six sentences, such that an entire team of differing academic disciplines understand the mission objectives and goals. The concept storytelling involves six sequential sentences (Fig. 6), and can be used to outline any mission:

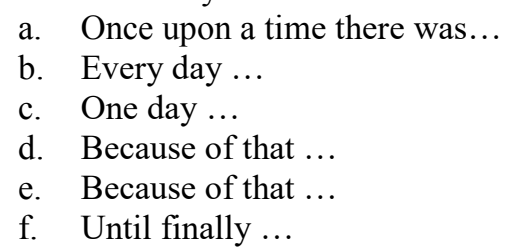

2. Introduction to the A-Team: The Architecture-Team at JPL consists of a group of people responsible for coming up with new mission proposals for NASA based on the mission constraints given to them. Specific topics taught by the A-Team included: cost analysis, science return diagraming, and science traceability matrices; key lessons used extensively throughout the week by the team [4].

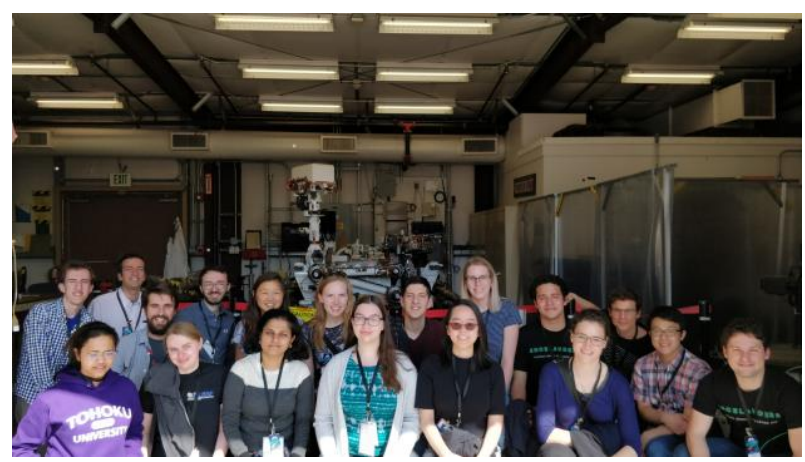

FIG 5: Team Voyager at NASA JPL Mars Yard

http://www.openculture.com/2013/03/pixars_22_rules_of_good_storytelling.h tml
In addition to the mission design-based workshops, team building and management presentations were also included on the opening night of the Challenge. Nigel Angold, of Angold Consulting, provided valuable insight into the structure of a good team, and the required phases each team must go through in order to be successful. Nigel was a continuous support system for Team Voyager, and assisted with disagreements in a non-judgmental, objective way. For Team Voyager, it was important to be able to have a third-party resource for conflict resolution, as will be discussed further in the Challenges section. Daily lectures related to space mission design, background science of Enceladus, and robotics engineering were hosted at Caltech with presenters from academia and industry. The lectures served as an additional knowledge resource to assist teams with their proposals, with time set aside at the end of each lecture for participants to ask the speakers questions.

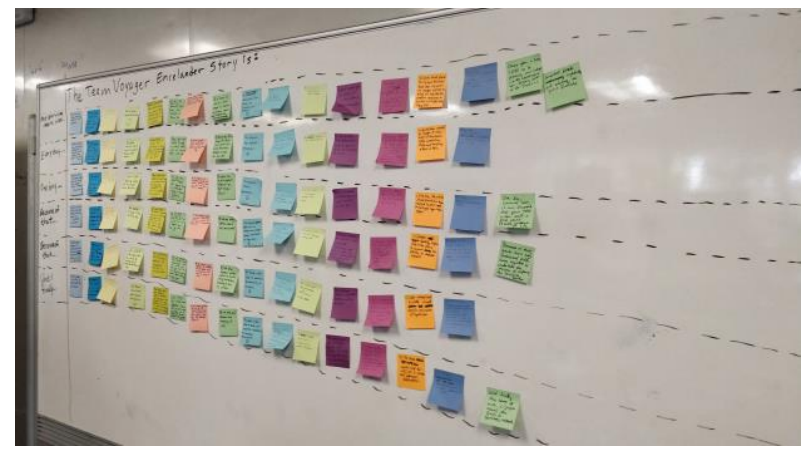

FIG 6: Team Voyager Storytelling using 6 sentence exercise

\section{Challenges}

Among the obvious challenge of coming up with a mission proposal in 5 days for a panel of the space industry's top professionals that Team Voyager overcame, there were many others. Miscommunication was a constant challenge to deal with, due to the unfamiliarity of the team members with one another at the conception of the week, the wide variety of academic backgrounds among team members, and the highstress environment. Systems engineers were delegated for the Science and Engineering teams and acted as a communication channel between the two teams, which assisted with deciphering what the Science team wanted to achieve and what solution the Engineering team could provide. Sub-team liaisons were extremely helpful for Team Voyager in managing conflict resolution between members, where the likely source of the disagreement arose from a misunderstanding.

The dependence on outside resources provided by the cochairs with respect to access to prominent researchers, industry experts and specific mission-related constraints proved to create difficulties for the team. As plans were being finalized, a new hypothetical risk would arise during discussions which caused setbacks prior to achieving clarification with outside sources. With an already limited 
amount of time, this led to sleepless nights in trying to achieve a plausible solution and completing the deliverables. Related to this, the dependency of each sub-team on one another and the need for concurrent engineering and design techniques required the constant use of the team liaisons to facilitate open communication when mission critical changes were made. An example of this in use was the late-week increased mission length proposed by the Science team, which required a reworking of the power budget by the engineering team to ensure enough power for the longer duration mission. The large team size also created an environment with 16 different opinions that needed to be given the opportunity to be shared. This not only extended the length of discussions in an already time-sensitive environment, but also led to many instances of opposing ideas, which would take time to reach a consensus between. In particular, the Science and Engineering sub-teams had to balance between what science objectives were critical to the mission to be accomplished, and what was possible to achieve with the engineering design. With miscommunication between sub-teams, rising tensions would then create situations where systems engineers would need to objectively take sides and prioritize based on budgeting, time, and mission objectives. Ideally, time must be managed in order to complete a task of this grandeur, and with many perspectives, it was difficult to come to a consensus and stick to the schedule. As mentioned, third-party mentors as well as the systems engineers took on the role of assisting with the management of constructive conversations, and with assurance from past years' participants, the team understood that the pace we were at was to be expected. Moreover, rather than framing the wide variety of opinions as detrimental to the team success, Team Voyager was able to truly consider every aspect of the design and scientific objective and came up with a well-thought-out mission concept with an abundance of evidence supporting why each choice was made. These opinions led to a thorough mission proposal that was ultimately selected as the winning option.

\section{Post-Challenge AND LeARning OUTCOMeS}

Following the challenge, participants were encouraged to share what they learned, and employ the techniques and tools utilized throughout the week in their academics and extracurriculars. Engineering team member Kelsey Doerksen ran a workshop for the University of Western Ontario's CubeSat team, based on the rapid brainstorming technique taught during the half-day JPL sessions. Undergraduate and graduate students were divided into pairs and given oneminute brainstorming sprints to come up with the components and required performance metrics for each of the CubeSat subsystems. Team member Julia Di, Engineering team member, has utilized techniques learnt at the JPL workshop during her robotics internship at JPL. Erica Nathan, Science team member, used the 6-sentence exercise with the undergraduate group in her lab, facilitating the activity to help with abstract writing. Yun-Hang Cho, team member of Explorer, spoke about his learning experience to his university. Similarly, Katiyayni Balachandran, systems team member for Voyager, presented on this challenge to her university through Students for the Exploration and Development of Space (SEDS) to encourage student participation in the future.

\section{CONCLUSION}

The Caltech Space Challenge is a rewarding, challenging, and unforgettable experience for all participants involved. It is a unique opportunity for students to get real, hands-on space mission design experience in a fast-paced environment with like-minded individuals. Participants can use what they have learnt throughout their academics in a practical way, to prepare them for future careers in space mission design. In particular, this year's topic inspired team members to continue research in icy moon exploration.

\section{ACKNOWLEDGEMENTS}

Team Voyager would like to thank the organizers of the 2019 Caltech Space Challenge, Simon Toedtli and Fabien Royer, for the amazing experience throughout the week. We would also like to thank Team Explorer, for pushing us to produce our best work, and for the friendly competition and comradery. Team Voyager would also like to thank all of the mentors, industry members, sponsors and volunteers, who took time to share their knowledge with the participants of the Space Challenge and make it an unforgettable week. The list of mentors include: Florian Kehl, Hayden Burgoyne, Ilana Gat, Jason Rabinovich, Kristina Hogstrom, and Kristjan Stone (NASA JPL), Nigel Angold (Angold Consulting), Thibaud Talon (Caltech), the speakers: David Murrow (Lockheed Martin), Jaakko Karras, Jean-Pierre de la Croix, Kalind Carpenter, Morgan Cable, Randii Wessen, Steve Matousek, Damon Landau, and Tom Nordheim (NASA JPL) and Thomas Heinsheimer (Aerospace). Finally, we would like to thank the panel of judges for their critical evaluation and for motivating us to consider all possibilities.

\section{REFERENCES}

[1] J.R. Wertz, D.F. Everett, J.J. Puschell, (2011), Space Mission Engineering: The New SMAD, Space Technology Library.

[2] NASA. (2005). Planetary protection provisions for robotic extraterrestrial missions. NPR $8020.12 C$.

[3] S.M. MacKenzie et al., (2016). THEO concept mission: Testing the habitability of Enceldus's Ocean. Advances in Space Research, 58(6), 1117-1137

[4] J.K. Ziemer, R. R. Wessen, and P. V. Johnson, (2016). Exploring the Science Trade Space with the JPL Innovation Foundry. NASA $J P L$ 